\title{
Apoio à Alocação de Recursos Humanos em Projetos de Software: Uma Abordagem Baseada em Satisfação de Restrições
}

\author{
Ahilton Silva Barreto ${ }^{1}$, Márcio de Oliveira Barros ${ }^{2}$, Claudia Maria Lima Werner ${ }^{1}$ \\ ${ }^{1}$ COPPE/UFRJ - Programa de Engenharia de Sistemas e Computação \\ Caixa Postal 68511 - CEP: 21945-970 \\ Rio de Janeiro - RJ - Brasil \\ ${ }^{2}$ DIA-UNIRIO \\ Av. Pasteur 458, Urca \\ Rio de Janeiro - RJ - Brasil \\ ahilton@cos.ufrj.br, marcio.barros@uniriotec.br, werner@cos.ufrj.br
}

\begin{abstract}
One of the main decisions that has to be made by the manager in a software project is how to staff the project, because the result of this activity is closely related to software quality. This work presents a support approach to staffing a software project. The required characteristics to perform activities, the characteristics possessed by each person and a set of constraints related to attributes such as cost, experience and team size will be considered. Based on this information, teams that satisfy the biggest amount of constraints and that eventually prioritize some selected factors will be suggested.
\end{abstract}

Resumo. Uma das principais decisões que precisa ser tomada pelo gerente em um projeto de software é como alocar pessoas a cada atividade do projeto, uma vez que essa alocação está fortemente ligada à qualidade do software. Este trabalho apresenta uma abordagem de apoio à alocação de pessoal em projetos de software. Serão consideradas características necessárias para a execução de cada atividade, as características possuídas por cada profissional e um conjunto de restrições associadas a fatores como custo, experiência e tamanho da equipe. A partir dessas informações serão sugeridas ao gerente alocações que satisfaçam ao maior número possível de restrições e, eventualmente, priorizem algum dos fatores selecionados.

\section{Introdução}

Apesar de já ser possível identificar melhorias importantes na taxa de sucesso de projetos de software, ainda há muito a ser feito para que aumente ainda mais o número de projetos bem sucedidos, ou seja, concluídos no prazo, no orçamento, com as funcionalidades e a qualidade desejadas [Standish Group 2003]. Uma das maneiras de aumentar esse número de projetos bem sucedidos é atentar para a gerência de projetos de software. Esta, quando inadequada, pode gerar diversos problemas no desenvolvimento de software, influenciando negativamente o sucesso dos projetos.

Em [PMI 2000], são descritas as áreas de conhecimento envolvidas na gerência de projetos. São elas as gerências de integração, de escopo, de tempo, de custo, de 
qualidade, de recursos humanos, de comunicações, de riscos e de aquisições do projeto. Dentre essas, iremos abordar a gerência de recursos humanos, mais especificamente, a alocação de recursos humanos em atividades que componham projetos de software.

As pessoas se comportam de forma não determinística e subjetiva, influenciando muito a produtividade no desenvolvimento de software, que é fundamentalmente uma atividade intelectual e social. No entanto, este é um dos fatores menos formalizado nos processos de desenvolvimento de software atuais, mesmo sendo evidente sua importância [Sommerville e Rodden 1995].

Na atividade de alocação de recursos humanos algumas decisões precisam ser tomadas pelo gerente de projetos. Entre elas, o gerente deve decidir quem alocar a cada atividade. Para isso, é preciso determinar quais as características (conhecimentos, habilidades, experiências, formação acadêmica e outras) necessárias para a realização de cada atividade, buscar na organização quais são os profissionais que possuem a qualificação necessária e que estejam disponíveis no momento e, finalmente, atribuir os profissionais às atividades a serem desempenhadas.

No entanto, essa tarefa não é simples, uma vez que geralmente há uma série de diferentes combinações de alocação possíveis. Por exemplo, considerando uma situação onde haja dez profissionais e dez atividades, serão dez bilhões de diferentes combinações. Portanto, realizar a atividade, considerando todos os fatores envolvidos e todas as combinações sem algum tipo de apoio, é extremamente difícil, ou até mesmo impossível. Devido a essa complexidade, o gerente nem sempre é capaz de considerar todas as equipes que podem ser montadas e o impacto de cada uma delas sobre algum fator (custo para o projeto, número de pessoas alocadas, entre outros) do projeto e acaba por escolher uma das equipes baseando-se apenas em sua experiência. No entanto, ao não considerar todas as potenciais equipes, há o risco de que o gerente não escolha a melhor equipe para uma dada situação. Assim, é interessante que o gerente seja apoiado nessa complexa atividade, de forma a reduzir o esforço necessário para sua realização e melhorar a qualidade das equipes escolhidas.

Neste trabalho é apresentada uma abordagem de apoio à realização da atividade de alocação de recursos humanos no planejamento de projetos de software. O objetivo é fornecer ao gerente de projetos um meio de realizar a atividade de forma mais rápida e precisa, sugerindo a ele possíveis equipes que satisfaçam a todas as restrições informadas por ele (características das pessoas e das atividades, períodos das atividades, custo, entre outros) e dando, também, ao gerente a possibilidade de obter equipes que satisfaçam a essas restrições e que representem as melhores soluções para algum fator envolvido no projeto, como por exemplo, a solução que represente o menor custo para o projeto ou a equipe com o menor número de profissionais.

A seção a seguir aborda a questão da importância da alocação de recursos humanos em projetos de software. A seção 3 aborda o apoio à decisão na Engenharia de Software e os problemas de satisfação de restrições, abordagem escolhida para a modelagem e solução do problema. A seção 4 apresenta a abordagem, a modelagem e solução propostas para o problema. A seção 5 apresenta a ferramenta construída para ilustrar a abordagem proposta. Finalmente, a seção 6 apresenta as considerações finais. 


\section{Gerência de Recursos Humanos}

De acordo com a norma NBR ISO 10006 (2000), são as pessoas que determinam a qualidade e o sucesso de um projeto. Portanto, fica clara a importância de escolher bem as pessoas que farão parte da equipe de um projeto de software.

As questões relativas a pessoas ganharam reconhecimento nos últimos anos como sendo centrais no gerenciamento de projetos de software. Dada a importância das pessoas no desenvolvimento de software, muitos estudos têm sido feitos buscando entender melhor como as pessoas se comportam, o que esperam, do que gostam e o que as faz trabalhar mais e melhor [Acuña e Juristo 2003][Agarwal e Ferrat 2000][Lee 2000].

Uma vez que as pessoas representam um fator tão determinante nos processos de desenvolvimento de software, atribuir tarefas adequadas à capacidade de cada profissional é de grande importância para que se possam atingir os níveis de produtividade e qualidade desejados em cada atividade. Atribuir pessoas com menos qualificações do que as exigidas pela atividade pode fazer com que esta não seja concluída, que seja concluída fora do prazo ou com baixa qualidade. Por outro lado, atribuir pessoas com qualificações muito maiores do que as exigidas pela atividade pode levar a um aumento desnecessário no custo do projeto ou até mesmo à frustração do profissional.

A gerência de recursos humanos de um projeto inclui os processos necessários para tornar mais efetivo o uso dos recursos humanos envolvidos no projeto [PMI 2000]. Está inserida na gerência de projetos, representando um item fundamental, dada a imensa importância das pessoas nos projetos, principalmente nos projetos de desenvolvimento de software.

Vários processos propostos na literatura contemplam, de alguma forma, a questão da gerência de recursos humanos. A norma NBR ISO 10006 (2000), que trata de diretrizes para a qualidade no gerenciamento de projetos, define os processos relacionados às pessoas. Esses processos visam criar um ambiente no qual a equipe possa contribuir efetiva e eficientemente para o projeto. Esses processos são os seguintes:

- Definição da estrutura organizacional do projeto: definir uma estrutura organizacional adequada às necessidades do projeto, incluindo a identificação de funções e definição de autoridade e responsabilidades;

- Alocação da equipe: selecionar e nomear pessoal suficiente, com competência apropriada para corresponder às necessidades do projeto;

- Desenvolvimento da equipe: desenvolver habilidades individuais e coletivas para melhorar o desempenho do projeto.

Dingsoyr e Royrvik (2001) permitem concluir, a partir da discussão sobre gerência de competências em uma organização, que um processo de gerência de recursos humanos consiste em três etapas principais:

- Busca das competências necessárias: inclui a definição das competências, a busca dos profissionais da organização que as possuem e a seleção dos profissionais desejados; 


\section{Simpósio Brasileiro de Qualidade de Software}

- Alocação de recursos humanos: inclui a associação de profissionais a tarefas;

- Desenvolvimento de competências: inclui a avaliação dos recursos humanos para posterior capacitação dos mesmos.

Em [Schnaider 2003], foi definido um processo de planejamento da alocação de recursos humanos. Nesse processo, devem ser definidos os perfis de competências que serão necessários à execução das várias atividades do projeto, visando à identificação de profissionais na organização que poderão ser alocados para executá-las. Essa atividade requer uma visão específica dos perfis de competência necessários à adequada execução de cada atividade do projeto, assim como uma visão global do perfil de competência de cada profissional da organização, sendo fortemente centrada na experiência e conhecimento do gerente do projeto.

Um outro aspecto importante que deve ser considerado na alocação de recursos humanos em um projeto de software é a criação de valor para o projeto e para a organização. O desenvolvimento de software envolve tempo, talento e dinheiro. Em um mercado competitivo, um dos maiores objetivos é maximizar a criação de valor para um dado investimento. Valor pode ser visto como time-to-market, reputação, qualidade do produto, entre outros. Assim sendo, o bom uso de cada recurso disponível é muito importante [Boehm e Sullivan 2000]. Como um dos recursos mais importantes na criação de valor para um projeto é justamente o recurso humano, a alocação deve não apenas considerar os aspectos técnicos (características necessárias para executar as atividades e características possuídas pelos profissionais), mas também deve ser feita de modo a priorizar algum fator que leve a criação de valor para o projeto [Barreto et al. 2005]. Exemplo desses fatores que podem variar de equipe para equipe são experiência da equipe, custo com a equipe do projeto, tamanho da equipe, entre outros.

\section{Apoio à Decisão Gerencial no Desenvolvimento de Software e Problemas de Satisfação de Restrições}

O desenvolvimento de software é um processo onde cada pessoa envolvida constantemente toma decisões, tanto técnicas quanto gerenciais. Um gerente de projetos tem um grande conjunto de fatores a considerar, várias atividades para planejar e controlar e diferentes decisões a tomar. Por exemplo: Como alocar tempo para as diferentes fases do projeto? Como alocar o pessoal? Como aumentar a qualidade do produto? Quanto de teste deve ser feito? Novas técnicas devem ser inseridas? O que funcionaria melhor para o projeto? [Rus et al. 2002].

Para responder a essas perguntas, um gerente poderia recorrer a diversas fontes. Há livros, artigos, publicações na Internet e grupos de discussão disponíveis, mas levaria muito tempo para investigar esses recursos e tempo é um recurso, particularmente, escasso para gerentes de projeto. Assim, seria interessante que houvesse formas de ajudá-los e guiá-los em suas decisões [Rus et al. 2002].

A disciplina de apoio à decisão na engenharia de software integra inteligência humana e computacional para facilitar a tomada de decisões melhores durante o ciclo de vida do software [Ruhe 2003]. A tomada de decisão é uma atividade importante e complexa na engenharia de software. Normalmente, é um processo não sistemático, uma vez que tipicamente se baseia em experiência pessoal, sem o uso de modelos explícitos [Rus et al. 2002]. 
O apoio à decisão na engenharia de software é de grande interesse tanto para a academia quanto para a indústria. Decisões precisam ser tomadas durante todas as iterações de um ciclo de vida de software. Atualmente, muitas dessas decisões cruciais são tomadas de forma ad hoc, baseadas em impressões e sem ligação com as melhores práticas, modelos e experiências [Ruhe 2003].

O apoio à decisão inteligente é útil, principalmente, em situações caracterizadas pelos seguintes fatores: complexidade, incerteza, presença de várias pessoas interessadas, grande quantidade de dados e problemas com parâmetros ou informações relacionadas que mudam rapidamente. Apoio, aqui, significa fornecer acesso à informação que seria de outra forma inacessível ou difícil de obter, facilitando a geração e avaliação de soluções distintas e priorizando alternativas através do uso de modelos explícitos que forneçam a estrutura para decisões particulares [Ruhe 2003].

Clemen e Reilly (2001) identificam algumas razões pelas quais as decisões são difíceis. Essas razões se aplicam ao desenvolvimento de software [Rus et al. 2002]:

- O desenvolvimento de software é um processo muito complexo, com muitas atividades, artefatos, atores e interações;

- Uma vez que é muito dependente das pessoas, o processo e seus produtos envolvem muita incerteza (pessoas saem, clientes mudam os requisitos, fornecedores não cumprem promessas, etc);

- Software deve ser construído mais rápido, mais barato e com maior qualidade;

- As pessoas tomam decisões de acordo com sua experiência, intuições, crenças pessoais e nas informações que têm disponíveis (que normalmente são imperfeitas, insuficientes ou imprecisas).

Muitos métodos e técnicas podem ser usados para auxiliar na modelagem de problemas de decisão. Entre esses, destacamos a modelagem dos problemas de decisão como problemas de satisfação de restrições [Russel e Norvig 2003].

Um problema de satisfação de restrições pode ser considerado como uma tupla $\mathrm{S}$ $=(\mathrm{V}, \mathrm{D}, \mathrm{R})$, onde $\mathrm{V}=\left\{\mathrm{x}_{1}, \mathrm{x}_{2}, \ldots, \mathrm{x}_{\mathrm{n}}\right\}$ é um conjunto finito de variáveis, também chamadas de variáveis de domínio finito, $\mathrm{D}=\left\{\mathrm{D}_{1}, \mathrm{D}_{2}, \ldots, \mathrm{D}_{\mathrm{n}}\right\}$ é um conjunto finito de domínios e R é um conjunto de restrições, limitando os valores que as variáveis podem receber simultaneamente. A cada variável $\mathrm{x}_{\mathrm{i}}$ está associado um conjunto finito $\mathrm{D}_{\mathrm{i}}$ com todos os possíveis valores que constituem seu domínio.

A solução de um problema de satisfação de restrições é uma atribuição de valores a todas as variáveis de $\mathrm{V}$, de forma que todas as restrições de $\mathrm{R}$ sejam satisfeitas. Diversos problemas, nas mais variadas áreas, são modelados e resolvidos como problemas de satisfação de restrições. Modelar um problema dessa forma tem a vantagem de possibilitar a utilização de todo o conhecimento que vem sendo adquirido ao longo dos anos sobre essa classe de problemas. Dessa forma, podem ser utilizados técnicas e algoritmos que têm sido propostos e avaliados para esse tipo de problema, sem a necessidade do desenvolvimento de novas técnicas e algoritmos específicos para um problema em questão. 


\section{Definição e Solução do Problema de Alocação de Recursos Humanos como um Problema de Satisfação de Restrições}

O problema da alocação de recursos humanos envolve, basicamente, um conjunto de profissionais e um conjunto de atividades. Cada profissional possui uma série de características (habilidades, conhecimentos, experiências, formação acadêmica, certificações, posição na organização, etc), cada qual com uma determinada intensidade (por exemplo, grande conhecimento em banco de dados, noções de linguagens de programação). Além disso, profissionais estão indisponíveis em diferentes períodos do tempo. Estar indisponível em um período significa que o profissional não pode ser alocado a nenhuma atividade de nenhum projeto naquele período. Essa indisponibilidade pode ser decorrente de diversos motivos, como férias, alocação a outra atividade no mesmo período, entre outros.

O custo da hora de trabalho do profissional e o número de horas trabalhadas por dia pelo profissional são também informações relevantes ao problema da alocação, uma vez que essas informações influem, respectivamente, no custo do projeto e na disponibilidade do profissional para alocação. Cada atividade, por sua vez, possui um conjunto de características exigidas, ou seja, quais características um profissional precisa possuir para executar a tarefa e, se relevante, em que intensidade. Além disso, uma atividade indica o período em que precisa ser realizada (informação esta, normalmente, contida no cronograma do projeto) e quantas horas por dia precisam ser dedicadas a sua execução nesse período.

Com base nestas informações, o problema da alocação de recursos humanos implica em atribuir um profissional a cada atividade, levando em consideração as seguintes regras:

- Para que um profissional seja atribuído a uma atividade, precisa possuir pelo menos todas as características exigidas pela atividade, em intensidade igual ou superior ao exigido;

- Para que um profissional seja atribuído a uma atividade, este não pode estar indisponível no período em que a atividade será executada;

- Um profissional está indisponível para realizar uma atividade:

o Se houver alguma indisponibilidade registrada para o período da atividade (férias, licença, viagem, etc);

o Se estiver alocado a outra atividade no período e o número de horas trabalhadas por dia nessa atividade for igual ao número máximo de horas trabalhadas por dia pelo profissional;

o Se estiver alocado a outra atividade no período e o número de horas trabalhadas por dia nessa atividade for menor que o número máximo de horas trabalhadas por dia pelo profissional, mas o número de horas restante não for suficiente para alocar o profissional a outra atividade no período. 


\section{Simpósio Brasileiro de Qualidade de Software}

Expressando de maneira formal:

- Seja $\mathrm{P}$ um conjunto de projetos. Cada elemento $\mathrm{P}_{\mathrm{i}} \in \mathrm{P}$ é composto por um nome e por um conjunto de atividades $\left(\mathrm{A}_{\mathrm{ij}}\right)$;

O $\mathrm{P}=\left\{\mathrm{P}_{\mathrm{i}}\right\}$

o $\quad P_{i}=\left[\right.$ nome, $\left.\left\{\mathrm{A}_{\mathrm{ij}}\right\}\right]$

- Seja RH um conjunto de profissionais da organização que podem ser alocados a atividades de seus projetos. Cada $\mathrm{RH}_{\mathrm{i}} \in \mathrm{RH}$ é composto por um nome, pelo valor da hora de trabalho, pela quantidade de horas trabalhadas por dia pelo profissional, por um conjunto de períodos de indisponibilidade $\left(\mathrm{PI}_{\mathrm{ij}}\right)$ e por um conjunto de características possuídas $\left(\mathrm{CRH}_{\mathrm{ij}}\right)$;

o $\quad \mathrm{RH}=\left\{\mathrm{RH}_{\mathrm{i}}\right\}$

o $\quad \mathrm{RH}_{\mathrm{i}}=$ [nome, \$hora, \#hora, $\left\{\mathrm{PI}_{\mathrm{ij}}\right\},\left\{\mathrm{CRH}_{\mathrm{ij}}\right\}$ ]

- Seja Ai um conjunto de atividades que pertence a um dado projeto $\mathrm{P}_{\mathrm{i}}$. Cada $A_{i j} \in A_{i}$ é composta por um nome, pelas datas inicial e final em que a atividade precisa ser realizada, pelo número de horas que devem ser dedicadas por dia à atividade e por um conjunto de características requeridas para que o profissional realize a atividade $\left(\mathrm{CA}_{\mathrm{ijk}}\right)$.

$0 \quad A_{i j}=\left[\right.$ nome, data_inicio, data_final, \#horas, $\left.\left\{\mathrm{CA}_{\mathrm{ijk}}\right\}\right]$

- Seja $\mathrm{PI}_{\mathrm{i}}$ um conjunto de períodos de indisponibilidade que pertence a um dado profissional $\mathrm{RH}_{\mathrm{i}}$. Cada $\mathrm{PI}_{\mathrm{ij}} \in \mathrm{PI}_{\mathrm{i}}$ é composto pelas datas inicial e final do período e pela quantidade de horas por dia no período em que o profissional estará indisponível.

$0 \quad \mathrm{PI}_{\mathrm{ij}}=$ [data_inicio, data_final, \#horas]

- Seja $\mathrm{C}$ um conjunto de características que pertence ou a um profissional $\mathrm{RH}_{\mathrm{j}}$ ou que são demandadas para a execução de uma atividade $A_{k}$. Cada $C_{i} \in C$ é composto por um nome, um valor máximo que a característica pode assumir e o valor (apresentado, no caso do profissional, ou demandado, no caso da atividade) da característica.

o $\mathrm{C}_{\mathrm{i}}=$ [nome, \#máximo, \#requerido/apresentado]

Assim, o problema pode ser descrito conforme mostrado a seguir:

$$
\forall A i j \in A i \subset P i \in P(\exists R H k \in R H(\alpha \wedge \beta))
$$

A formulação acima indica que para toda atividade que pertence ao conjunto de atividades de um determinado projeto, que por sua vez pertence ao conjunto de projetos da organização, existe algum profissional do conjunto de profissionais da organização para o qual as condições $\alpha$ e $\beta$ são verdadeiras. A primeira condição é a seguinte:

$$
\alpha \leftarrow \forall\left(\left(C m \in C \subset A_{i}\right)\left(\exists\left(\left(C l \in C \subset R H_{k}\right) \wedge C m=C l \wedge \operatorname{Valor}(C l) \geq \operatorname{Valor}(C m)\right)\right)\right)
$$

A fórmula acima estabelece que, para toda característica que faz parte do conjunto de características exigidas por uma atividade, existe uma característica que faz parte do conjunto de características possuídas por um profissional, de forma que as duas 


\section{Simpósio Brasileiro de Qualidade de Software}

características são, na verdade, a mesma e o valor possuído da característica no profissional é maior ou igual ao valor exigido na atividade.

A segunda condição expressa na primeira fórmula é a seguinte:

$$
\beta \leftarrow \neg \exists\left(P I_{n} \in P I \subset R H_{k}\right)\left(\begin{array}{l}
\left(\operatorname{DataIni}\left(A_{i}\right) \geq \operatorname{DataIni}\left(P I_{n}\right)\right) \wedge \\
\left(\operatorname{DataIni}\left(A_{i}\right) \leq \operatorname{DataFim}\left(P I_{n}\right)\right) \\
\vee \\
\left(\operatorname{DataFim}\left(A_{i}\right) \geq \operatorname{DataIni}\left(P I_{n}\right)\right) \wedge \\
\left(\operatorname{DataFim}\left(A_{i}\right) \leq \operatorname{DataFim}\left(P I_{n}\right)\right) \\
\vee \\
\left(\operatorname{DataIni}\left(A_{i}\right) \leq \operatorname{DataIni}\left(P I_{n}\right)\right) \wedge \\
\left(\operatorname{DataFim}\left(A_{i}\right) \geq \operatorname{DataFim}\left(P I_{n}\right)\right)
\end{array}\right)
$$

A expressão acima estabelece que não existe um período de indisponibilidade para o profissional tal que o período de indisponibilidade esteja compreendido todo, ou em parte, dentro do período em que a atividade precisa ser feita. E que, além disso, o número máximo de horas trabalhadas por dia pelo profissional seja menor que o número de horas de indisponibilidade por dia somado ao número de horas que devem ser dedicados por dia à atividade.

Conforme dito na seção 3, um problema de satisfação de restrições pode ser considerado como uma tupla $\mathrm{S}=(\mathrm{V}, \mathrm{D}, \mathrm{R})$. Dessa forma, pode-se modelar o problema da alocação de recursos humanos em projetos de software como sendo um problema de satisfação de restrições, no qual as atividades são modeladas como variáveis, os valores que essas variáveis podem assumir são os profissionais da organização e todas as regras envolvidas (de qualificação e de data) são as restrições. Resolver o problema implica em escolher profissionais (valores) para todas as atividades (variáveis), de forma a satisfazer todas as regras definidas para o problema (restrições).

Dessa forma, pode-se considerar R como sendo as regras expressas nas equações acima. V e D podem ser definidos formalmente da seguinte maneira:

$$
\begin{aligned}
& \mathrm{D}=\left\{\mathrm{D}_{\mathrm{i}}\right\} \\
& \mathrm{D}_{\mathrm{i}}=\left[\left\{\mathrm{RH}_{\mathrm{ij}}\right\}\right] \\
& \mathrm{V}=\left\{\mathrm{V}_{\mathrm{i}}\right\} \\
& \mathrm{V}_{\mathrm{i}}=\left[\mathrm{A}_{\mathrm{ij}}, \mathrm{D}_{\mathrm{ij}}\right]
\end{aligned}
$$

Normalmente, há várias soluções para um problema de satisfação de restrições. Elas têm diferentes qualidades, definidas por funções de custo. Em muitos problemas, interessa uma solução ótima, que minimiza ou maximiza sua função de custo. No problema da alocação de recursos humanos em projetos de software podem ser definidas algumas funções de custo com base em fatores do problema. Cada função de custo terá uma solução ótima própria, potencialmente distinta das soluções ótimas geradas para outras funções de custo. Neste trabalho são consideradas as seguintes funções: 


\section{Simpósio Brasileiro de Qualidade de Software}

- Menor Custo: esta função é responsável por determinar a alocação que satisfaz a todas as restrições do problema e, além disso, representa o menor custo para o projeto. O custo é calculado a partir do valor da hora de trabalho dos profissionais alocados, multiplicado pelo número de horas que devem ser dedicadas às atividades do projeto;

- Menor Equipe: esta função é responsável por determinar a alocação que satisfaz a todas as restrições do problema e, além disso, representa a equipe em que menos profissionais estão alocados ao projeto;

- Menor Sub-Alocação: esta função é responsável por determinar a alocação que satisfaz a todas as restrições do problema e, além disso, representa a equipe em que há o menor "índice de sub-alocação" no projeto. Esse índice é baseado no valor numérico dos valores das características exigidas pelas atividades e possuídas pelos profissionais. Cada valor de característica possuído pelo profissional que excede o valor exigido pela atividade soma essa diferença no índice. É um índice relativo, uma vez que nem todas as características possuem o mesmo valor máximo;

- Maior Qualificação: esta função é responsável por determinar a alocação que satisfaz a todas as restrições do problema e, além disso, representa a equipe em que as melhores pessoas disponíveis foram alocadas. Nesta função, utiliza-se o mesmo índice de sub-alocação, só que, ao invés de selecionar a equipe com o menor índice, é selecionada a equipe com o maior valor para esse índice, ou seja, a maior sub-alocação.

Até o momento, foi considerado o caso em que existe pelo menos uma solução para o problema. No entanto, em muitas aplicações importantes as restrições podem ser muito fortes, de forma que uma solução completa não seja possível. Nesse caso, soluções parciais ainda podem ser úteis se um número suficiente das restrições mais importantes for satisfeito. No caso do problema da alocação de recursos humanos em projetos de software essa situação pode ocorrer, ou seja, as restrições das atividades não poderem ser satisfeitas pelos profissionais disponíveis na organização. Neste caso, nem sempre a organização irá optar pela contratação ou capacitação de profissionais e irá, na verdade, procurar atender ao máximo possível de restrições. Assim, no problema da alocação, caso não haja uma solução que satisfaça a todas as restrições, uma abordagem é retornar a alocação que quebre o menor número possível de restrições. Neste trabalho essa abordagem foi escolhida. No entanto, deu-se também a possibilidade de retornar a solução parcial que teve profissionais alocados ao maior número possível de atividades sem quebrar nenhuma restrição.

Assim sendo, a abordagem proposta para a solução do problema da alocação de recursos humanos neste trabalho contempla diferentes possibilidades. São elas:

- Apenas uma solução: retornar uma solução qualquer que satisfaça às restrições;

- Todas as soluções: retornar todas as soluções que satisfaçam às restrições;

- Problema ótimo: retornar as soluções que satisfaçam às restrições e que representem as soluções de melhor custo dado um fator (menor custo, menor equipe, menor sub-alocação, melhor qualificação); 
- Problema parcial ótimo quanto às restrições: retornar as soluções parciais que quebrem o menor número de restrições do problema;

- Problema parcial ótimo quanto às atividades: retornar as soluções parciais que atribuam profissionais ao maior número de atividades.

\section{Exemplo de Utilização da Técnica Proposta}

Para possibilitar um melhor entendimento da técnica de alocação proposta neste trabalho, nesta seção será apresentado um exemplo de sua utilização. Será definida uma situação com um projeto, um conjunto de atividades e profissionais fictícios e serão mostradas as possíveis soluções para o problema, dadas as restrições impostas.

Sejam, portanto, as seguintes características e seus domínios:

Tabela 1. Características do exemplo e seus domínios

\begin{tabular}{|l|c|l|}
\hline \multirow{2}{*}{\multicolumn{1}{|c|}{ Características }} & \multicolumn{2}{c|}{ Domínios } \\
\cline { 2 - 3 } & $\begin{array}{c}\text { Valor } \\
\text { Numérico }\end{array}$ & \multicolumn{1}{c|}{ Descrição } \\
\hline $\begin{array}{l}\text { Relacionamento com o Público } \\
\text { Negociação } \\
\text { Trabalho em Equipe }\end{array}$ & 1 & Tem treinamento \\
\cline { 2 - 3 } $\begin{array}{l}\text { Técnicas de Lev. de Requisitos } \\
\text { Análise Orientada a Objetos } \\
\text { Banco de Dados } \\
\text { Projeto Orientado a Objetos }\end{array}$ & 2 & Possui Habilidade \\
\cline { 2 - 3 } $\begin{array}{l}\text { Java } \\
\text { Técnicas de Testes }\end{array}$ & 1 & Possui Grande Habilidade \\
\hline & 2 & Conhece e Sabe Executar com Supervisão \\
\cline { 2 - 3 } & 3 & Possui Expertise e Mentoriza \\
\hline Experiência em Telecomunicações & 1 & Entre 2 e 6 meses \\
\cline { 2 - 3 } & 2 & Entre 6 meses e 1 ano \\
\cline { 2 - 3 } & 3 & Entre 1 e 3 anos \\
\hline
\end{tabular}

A tabela 2 apresenta as atividades e os profissionais utilizados no exemplo. Cada atividade é apresentada com seu nome e período em que deve ser realizada. Abaixo da linha com o nome da atividade é exibida a lista das características exigidas, juntamente com o valor numérico da intensidade da característica que o profissional deve possuir para realizar a atividade. Os profissionais são apresentados nas colunas, que contêm também o valor da hora de trabalho do profissional. São indicados então, para cada característica exigida pelas atividades, a intensidade possuída pelo profissional naquela característica. As regiões sombreadas indicam, considerando somente as restrições de características exigidas, que o profissional pode executar a atividade. 
Tabela 2. Atividades e Profissionais do Exemplo

\begin{tabular}{|c|c|c|c|c|c|c|c|c|}
\hline \multirow{2}{*}{\multicolumn{2}{|c|}{ Profissional }} & Prof. 1 & Prof. 2 & Prof. 3 & Prof. 4 & Prof. 5 & Prof. 6 & Prof. 7 \\
\hline & & $15,00 \mathrm{~h}$ & $30,00 \% \mathrm{~h}$ & $50,00 \mathrm{~h}$ & $20,00 / \mathrm{h}$ & $10,00 \mathrm{~h}$ & $15,00 \mathrm{~h}$ & $75,00 \mathrm{ih}$ \\
\hline Especificaçẫo de Requisitos 22/03/05 & $29 / 03 / 05$ & & & & & & & \\
\hline Relacionamento com o Público & 2 & & 2 & 3 & & & & 3 \\
\hline Negociaçẫo & 2 & 2 & 2 & 3 & & 2 & & 3 \\
\hline $\begin{array}{l}\text { Técnicas de Levantamento de } \\
\text { Requisitos }\end{array}$ & 2 & & 2 & & 2 & & & \\
\hline $\begin{array}{l}\text { Experiência em } \\
\text { Telecomunicaçốes }\end{array}$ & 2 & & 3 & 3 & & & & 4 \\
\hline Análise do Sistema & $07 / 04 / 05$ & & & & & & & \\
\hline Trabalho em Equipe & 2 & 2 & 2 & 3 & 2 & 2 & & 3 \\
\hline Análise Orientada a Objetos & 2 & & 2 & & 3 & & & 3 \\
\hline Projeto do Sistema & $14 / 04 / 05$ & & & & & & & \\
\hline Trabalho em Equipe & 2 & 2 & 2 & 3 & 2 & 2 & & 3 \\
\hline Banco de Dados & 2 & 2 & 2 & 3 & 2 & 2 & 2 & 3 \\
\hline Projeto Orientado a Objetos & 2 & & 2 & 3 & & & & 3 \\
\hline Construção do Sistema & $30 / 04 / 05$ & & & & & & & \\
\hline Banco de Dados & 2 & 2 & 2 & 3 & 2 & 2 & 2 & 3 \\
\hline Java & 2 & 2 & & 3 & 2 & 2 & 3 & 3 \\
\hline Testes do Sistema & $06 / 05 / 05$ & & & & & & & \\
\hline Técnicas de Testes & 2 & 2 & & 2 & 2 & & & \\
\hline Java & 2 & 2 & & 3 & 2 & 2 & 3 & 3 \\
\hline
\end{tabular}

Considerando-se as atividades e os profissionais descritos na tabela 2 é possível montar 84 diferentes equipes que atendam às restrições. Se o simples fato de determinar uma equipe que atendesse aos requisitos impostos sem nenhum apoio já seria difícil, mais difícil ainda seria gerar essas 84 equipes calculando fatores como custo, tamanho e sub-alocação dos profissionais em cada uma delas. Vale salientar que se fossem retiradas as características exigidas das atividades e não houvesse sobreposição de atividades, seriam possíveis mais de 16800 alocações diferentes.

Neste exemplo, as funções de custo definidas para o problema variam da seguinte forma:

Tabela 3. Variação dos fatores no Exemplo

\begin{tabular}{|l|c|c|}
\hline Fator & $\begin{array}{c}\text { Valor } \\
\text { Mínimo }\end{array}$ & $\begin{array}{c}\text { Valor } \\
\text { Máximo }\end{array}$ \\
\hline Custo & $7.760,00$ & $22.920,00$ \\
\hline Tamanho da Equipe & 2 & 5 \\
\hline $\begin{array}{l}\text { Índice de Sub- } \\
\text { Alocação }\end{array}$ & 75 & 165 \\
\hline
\end{tabular}

Com isso, percebe-se que há uma grande variação em todos os fatores considerados. Uma vez que o gerente dificilmente seria capaz de considerar todas as possibilidades e otimizar esses fatores sem apoio, fica claro o quanto se pode desviar de uma solução ótima.

Exemplificando, caso o objetivo do gerente seja determinar uma equipe que atenda às restrições e procure diminuir os gastos do projeto, é possível que ele, sem apoio, se distancie da equipe que representa o menor custo em até $15.160,00$ e a solução 


\section{Simpósio Brasileiro de Qualidade de Software}

de maior custo representa quase três vezes o valor da solução de menor custo. Já se o objetivo for diminuir o tamanho da equipe, é possível encontrar equipes com dois profissionais até equipe com cinco profissionais (duas vezes e meia maior que a menor equipe possível).

Assim, é possível ilustrar o quanto é difícil considerar todas as opções e satisfazer a todas as restrições de forma $a d-h o c$, e o quanto se pode distanciar de um valor ótimo para algum fator envolvido no problema, mesmo em um exemplo que representa um projeto pequeno, com poucas pessoas e atividades, além da curta duração.

\section{Ferramenta de Apoio}

Para demonstrar o uso da técnica proposta foi implementada uma ferramenta de apoio que fornece sugestões de possíveis equipes em cada situação. O objetivo da ferramenta é permitir que o gerente informe os dados das atividades do projeto, dos profissionais e as restrições envolvidas (características, datas, indisponibilidades) e escolha uma das opções de montagem de equipe implementadas:

- Uma ou todas as soluções (no entanto, a escolha de todas as soluções é desaconselhada devido à possibilidade de retornar bilhões de soluções);

- Otimizar ou não algum fator. Caso se deseje otimizar, selecionar o fator;

- Gerar ou não soluções parciais, caso não seja possível satisfazer todas as restrições.

De acordo com as opções escolhidas, diferentes algoritmos são utilizados para gerar as equipes que atendam a essas opções. Vale salientar que todas as possíveis combinações são consideradas, o que seria talvez impossível sem o apoio computacional.

A ferramenta, então, exibe as possíveis combinações de equipe de acordo com as opções escolhidas, trazendo para cada equipe o valor de cada fator calculado para a equipe (custo, tamanho, sub-alocação). Os algoritmos para a geração das equipes utilizam diversas técnicas, tais como branch and bound, backtracking, forward checking, most constrained variable, partial constraint satisfaction, entre outras [Russel e Norvig 2003]. A linguagem de programação utilizada foi Java.

A ferramenta foi implementada de modo a poder ser usada isoladamente, mas também ser integrada a outras ferramentas através de arquivos XML com os dados necessários. Os dados das equipes geradas também podem ser exportados via XML.

Na figura 1 é mostrada uma das telas da ferramenta, a tela de cadastro de profissionais. Nesta tela são informados os dados do profissional, incluindo os períodos de indisponibilidade e as características possuídas por ele. 


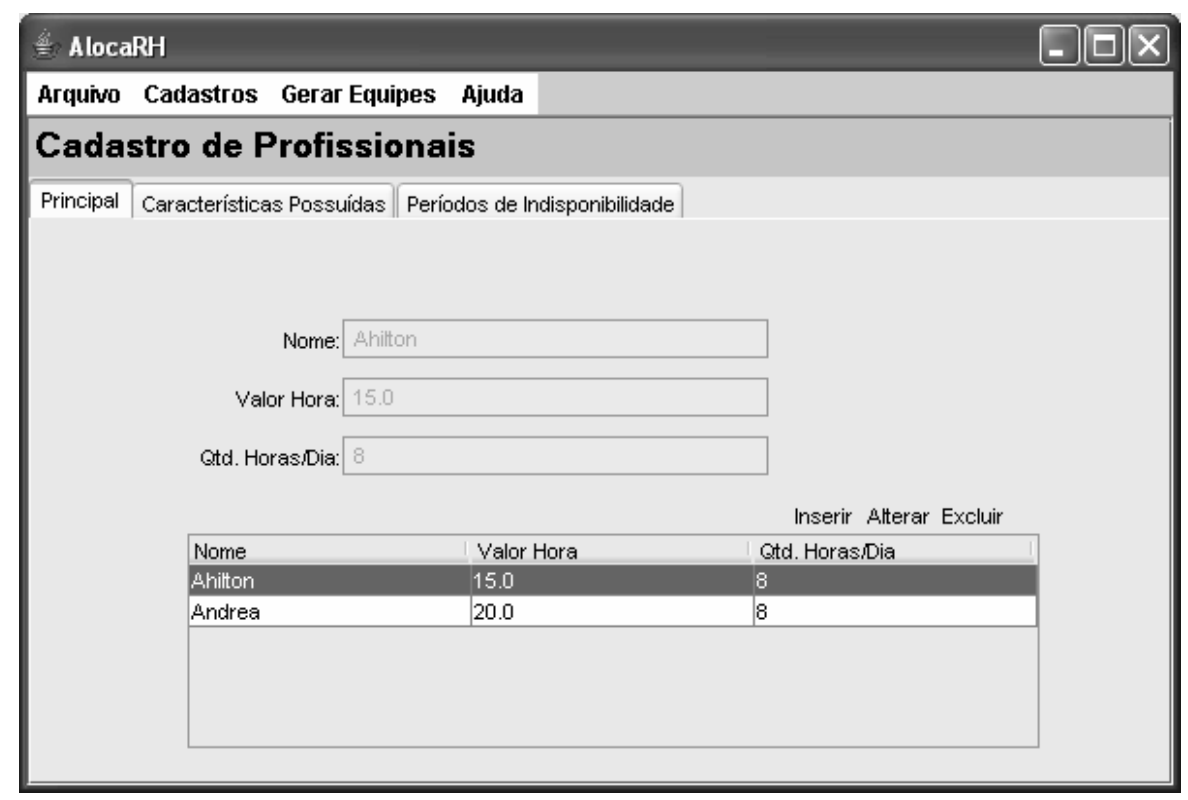

Figura 1. Cadastro de Profissionais

Na figura 2 é mostrada a tela de geração de equipes. Nesta tela são informados os parâmetros dos algoritmos de alocação e são mostradas as características das equipes geradas. Os detalhes de cada equipe (atividades, profissionais, datas) são mostrados em "Detalhes da Equipe".

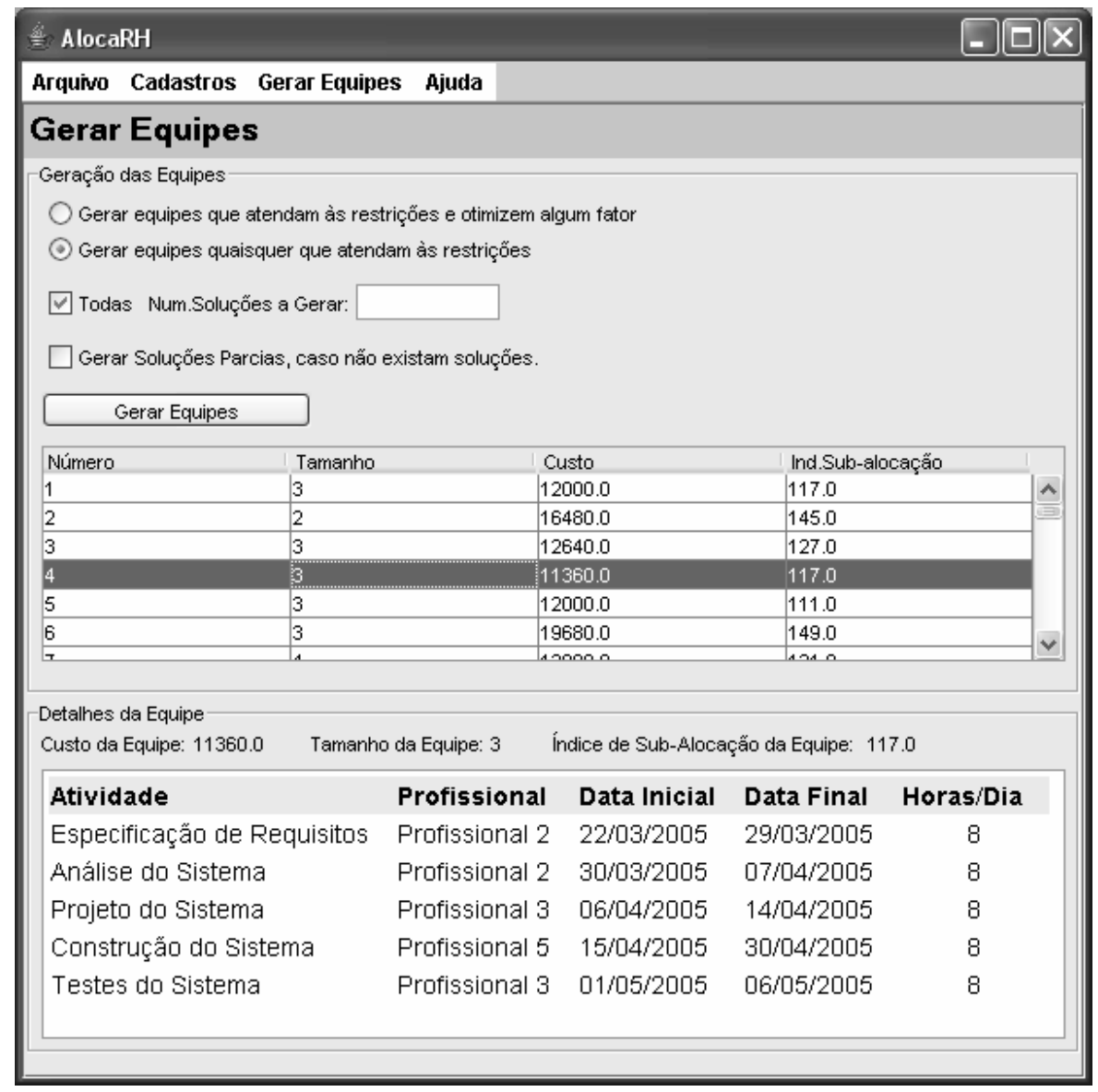

Figura 2. Tela de Geração de Equipes 


\section{Considerações Finais}

Este trabalho apresentou uma abordagem de apoio à alocação de recursos humanos em projetos de desenvolvimento de software baseada na satisfação de restrições. Esta representa uma maneira de auxiliar o gerente de projetos nessa atividade complexa e importante no desenvolvimento de software.

A abordagem proposta representa um exemplo do uso de técnicas de otimização e inteligência artificial para apoiar a execução de atividades complexas do processo de desenvolvimento de software. Abordagens similares poderiam ser usadas para apoiar outras atividades, tais como seleção de fornecedores, seleção de componentes para reutilização, escolha da arquitetura de sistemas, escolha de modelos de ciclo de vida, entre outras.

A abordagem considera, de certa forma, a preocupação tanto com questões técnicas (quando considera as características) quanto com os objetivos do negócio (quando prioriza fatores como custo e tamanho de equipe). Da mesma forma, abordagens similares poderiam ser adotadas em outras atividades do processo de desenvolvimento de software, de forma que na execução de cada tarefa houvesse uma maior preocupação com a integração entre as questões técnicas e os objetivos do negócio.

A principal perspectiva futura deste trabalho é a realização de um estudo de caso para avaliar a abordagem proposta. O estudo está em fase de planejamento e terá como objetivo avaliar o tempo de execução da atividade de seleção da equipe e a qualidade das equipes geradas pela aplicação da abordagem proposta, em comparação com a realização da atividade de maneira $a d-h o c$. Além disso, podem ainda ser citadas como perspectivas futuras a integração da ferramenta desenvolvida com ambientes de desenvolvimento de software e a definição de outras funções de utilidade que estejam envolvidas no problema.

\section{Referências}

Acuña, S.T. e Juristo, N. (2003) "Modelling Human Competencies in the Software Process", ProSim'03, Portland, Estados Unidos.

Agarwal, R. e Ferrat, T.W. (2000) "Retention and the career motives of IT professionals", Proceedings of the 2000 ACM SIGCPR conference on Computer personnel research.

Barreto, A.S., Barros, M.O., Werner, C.M.L. (2005) "Staffing a Software Project: a Constraint Satisfaction Approach", 7th International Workshop on EconomicsDriven Software Engineering Research, St. Louis, Estados Unidos.

Boehm, B. e Sullivan, K. (2000) "Software economics: a roadmap" In: The Future of Software Engineering, 22nd International Conference on Software Engineering, pp. 319-344.

Clemen, R. e Reilly, T. (2001) Making Hard Decisions With Decision Tools, Duxbury Thomson. 
Dingsoyr e Royrvik (2001), "Skills Management as Knowledge Technology in a Software Consultancy Company”, K. - D. Althoff, R.L. Feldmann, and W.Müller (Eds.): LSO 2001, LNCS 2176, pp. 96-103.

Lee, P.C.B. (2002) "The social context of turnover among information technology professionals”, Proceedings of the 2002 ACM SIGCPR conference on Computer personnel research.

NBR ISO 10006 (2000), “Gestão da Qualidade - Diretrizes para a Qualidade no Gerenciamento de Projetos”, Associação Brasileira de Normas Técnicas, Rio de Janeiro, RJ, Brasil.

PMI (2000) “PMBOK - Project Management Body of Knowledge”, Project Management Institute, 2000.

Ruhe, G. (2003) "Software Engineering Decision Support”, ICORE Research Report, Vol. 2, pp. 153-162.

Rus, I., Biffl, S. e Halling, M. (2002) "Systematically Combining Process Simulation and Empirical Data in Support of Decision Analysis in Software Development", SEKE, Itália, Julho 2002, pp.827-833.

Russell, S. e Norvig, P. (2003) “Artificial Intelligence: A Modern Approach”, cap. 4, pp. 92-119, 2nd Edition, Prentice Hall.

Schnaider, L.R.C. (2003), "Planejamento da alocação de recursos humanos em ambientes de desenvolvimento de software orientados à organização", Tese de M. Sc., COPPE/UFRJ, Rio de Janeiro, RJ, Brasil.

Sommerville, I e Rodden, T. (1995), "Human, social and organizational influences on the software process", Lancaster University, Computing Department, Cooperative Systems Engineering Group, Technical Report: CSEG/2/1995, 1995, 1-21.

Standish Group (2003), "Latest Standish Group CHAOS Report Shows Project Success Rate Have Improved By 50\%”, Online: http://www.standishgroup.com /press/article.php?id=2 (verificado em fevereiro, 2004). 